\title{
Equations paraboliques d'ordre élevé : représentations analytiques de solutions numériques
}

\section{J. PIRAUX}

CNRS, Laboratoire de Mécanique et d'Acoustique, 31 Chemin Joseph Aiguier, 13402 Marseille cedex 9, France

\begin{abstract}
A parabolic wave equation is etablished with a particular approximation by rational fraction of degree $N$. An analytical solution is given for this approximate equation, in the case of a homogeneous medium of constant depth with a free surface and a rigid bottom. An analytical representation is also obtained for the numerical solution of this equation by the alternating direction implicit (ADI) method. The advantages of this method over the usual ones are indicated.
\end{abstract}

\section{Introduction.}

Dans un papier présenté il y a deux ans [1], on a écrit une équation d'onde de type parabolique associée à une approximation de l'opérateur $(1+L)^{1 / 2}$, où $L$ est "suffisamment petit", par une fraction rationnelle en $L$ quelconque de degré $N$. On généralisait ainsi les travaux de Knightly et al. [2] (fraction rationnelle de degré 2). Pour l'équation d'onde exacte, pour l'équation d'onde approchée, et pour la résolution numérique de cette équation approchée à l'aide de la méthode de Crank-Nicholson, on a écrit des solutions analytiques valables pour un milieu homogène de profondeur constante avec un fond parfaitement réfléchissant. On généralisait ainsi les travaux de Vefring et Mjølsnes [3] (solution analytique avec $N=3$ pour l'équation d'onde approchée seulement, non résolue numériquement).

Une approximation moins générale de $(1+L)^{1 / 2}$ a été développée par Bamberger et al. [4] et utilisée par Collins [5]. La méthode de résolution numérique adaptée est la méthode des directions alternées implicite (ADI). Dans les conditions de propagation énoncées ci-dessus, on peut encore donner une solution analytique pour le problème approchée et pour ce problème résolu numériquement. Cette dernière solution permet de comparer les deux méthodes ffraction quelconque de degré $N+$ méthode de Crank-Nicholson\} et $\{$ "fraction de Bamberger" + méthode ADI . 


\section{L'approximation de Bamberger et la méthode ADI.}

L'équation d'onde de type parabolique est (voir [1] à [3]) :

$$
\frac{\partial u}{\partial r}+i k_{0}\left\{1-(1+L)^{1 / 2}\right\} u=0
$$

où la fonction $u(r, z)$ et la pression acoustique $p(r, z)$ sont reliées par $p(r, z)=H_{0}^{(1)}\left(k_{0} r\right) u(r, z) . k_{0}$ est un nombre d'onde de référence et $L$ est l'opérateur défini par :

$$
L=\left(\nu^{2}-1\right)+\frac{1}{k_{0}^{2}} \frac{\partial^{2}}{\partial z^{2}}
$$

$\nu$ est un indice de réfraction, rapport du nombre d'onde $k$ en un point du milieu, donc fonction des coordonnées de ce point, au nombre d'onde de référence $k_{0}$.

Bamberger et al. [4] approchent l'opérateur pseudo-différentiel $(1+L)^{1 / 2}$ par une somme de fractions élémentaires du premier degré :

$$
(1+L)^{1 / 2} \approx 1+\sum_{j=1}^{N} \frac{a_{j} L}{1+b_{j} L}
$$

L'équation d'onde approchée obtenue est :

$$
\frac{\partial u}{\partial r}=i k_{0} \sum_{j=1}^{N} \frac{a_{j} L}{1+b_{j} L} u
$$

La méthode des directions alternées implicite consiste, pour passer de la valeur de $u$ calculée en $(r, z)$ à la valeur de $u$ calculée en $(r+\Delta r, z)$, à résoudre successivement $N$ problèmes élémentaires :

$$
\frac{\partial u}{\partial r}=i k_{0} \frac{a_{j} L}{1+b_{j} L} u \quad(j=1, \ldots, N)
$$

Chaque problème élémentaire est résolu par la méthode de Crank-Nicholson.

\section{Une solution analytique.}

Pour l'équation (2), et dans le cas d'un milieu homogène de profondeur constante $B$ avec un fond parfaitement réfléchisssant, donc avec les conditions aux limites $u(r, 0)=0$ et $\partial u / \partial z(r, B)=0$, la solution analytique peut s'écrire sous la forme d'une somme de modes : 


$$
v_{n}(r, z)=\sin \zeta_{n} z \exp \left\{i k_{0} B_{n} r\right\}
$$

avec : $\quad \zeta_{n}=\left(n+\frac{1}{2}\right) \frac{\pi}{B}, \quad B_{n}=\sum_{j=1}^{N} \frac{a_{j} x_{n}^{j}}{1+b_{j} x_{n}^{j}}, \quad x_{n}=-\frac{\zeta_{n}^{2}}{k_{0}^{2}}$.

C'est la même solution que celle donnée dans le papier précédent, mais réécrite pour la nouvelle forme de la fraction rationnelle.

Pour l'équation résolue numériquement par la méthode ADI avec des pas de discrétisation $\Delta r$ et $\Delta z$ en distance et en profondeur respectivement, la solution peut aussi s'écrire sous la forme d'une somme de modes :

$$
w_{n}(r, z)=\sin \zeta_{n} z \exp \left\{i \frac{\tan ^{-1}\left(k_{0} C_{n} \Delta r / 2\right)}{\Delta r / 2} r\right\}
$$

avec :

$$
y_{n}=x_{n}\left\{\frac{\sin \frac{\zeta_{n} \Delta z}{2}}{\frac{\zeta_{n} \Delta z}{2}}\right\}^{2} \quad, \quad C_{n}=\frac{2}{i k_{0} \Delta r} \frac{\pi_{n}^{+}-\pi_{n}^{-}}{\pi_{n}^{+}+\pi_{n}^{-}}
$$

et :

$$
\pi_{n}^{-}=\prod_{j=1}^{N}\left\{1-\frac{i k_{0} \Delta r}{2} \frac{a_{j} y_{n}}{1+b_{j} y_{n}}\right\} \quad, \quad \pi_{n}^{+}=\prod_{j=1}^{N}\left\{1+\frac{i k_{0} \Delta r}{2} \frac{a_{j} y_{n}}{1+b_{j} y_{n}}\right\}
$$

L'expression de $w_{n}(r, z)$ ressemble à celle obtenue dans le papier précédent. Il n'en est pas de même pour l'expression de $C_{n}$, ce qui confère de nouvelles propriétés à l'algorithme utilisé ici.

On remarque que la dépendance en $z$ des solutions élémentaires (3) et (4) est la même que la dépendance des solutions élémentaires exposées dans le papier précédent [1], la même aussi que celle des solutions élémentaires de l'équation de départ, l'équation de Helmholtz, avec l'approximation de champ lointain. C'est le résultat bien connu, selon lequel la méthode de l'équation parabolique n'affecte pas l'amplitude d'un mode, mais seulement son argument.

\section{Avantages de la méthode $\{$ "fraction de Bamberger" + ADI\}.}

La méthode $\{$ "fraction de Bamberger" + ADI\} présente des avantages sur la méthode classique \{fraction quelconque de degré $N+$ Crank-Nicholson\}. On en énonce trois. Les deux premiers sont connus. Le troisième est nouveau. 
- Il n'y a pas à calculer les puissances de l'opérateur $L$ : c'est un gain considérable, il suffit de calculer $L^{3}$ à la main pour en être convaincu. Ne pas calculer $L^{n}$ signifie aussi ne pas avoir à évaluer de dérivées de l'indice de réfraction $\nu$ par rapport à la profondeur et ne pas avoir à écrire des conditions aux limites supplémentaires pour les dérivées d'ordre élevé de $u$ à la surface libre et au fond de la couche liquide.

- La méthode de Crank-Nicholson et une fraction rationnelle de degré $N$ conduisent à la résolution de systèmes linéaires avec des matrices bandes à $2 N+1$ "diagonales". La méthode ADI et la fraction rationnelle utilisée par Bamberger et al. [4] conduisent à la résolution de $N$ systèmes linéaires avec des matrices qui sont toujours tridiagonales, ce qui nécessite un minimum de temps machine et d'encombrement mémoire.

- Dans le cas de la méthode $\{$ "fraction de Bamberger" + ADI\}, on montre, grâce à la solution analytique (4), qu'il est possible d'augmenter le degré $N$ (par exemple en le doublant) en réduisant le nombre de pas d'intégration en distance (c'est-à-dire, dans l'exemple précédent, en doublant le pas d'intégration $\Delta r$ ) sans modifier l'erreur d'intégration, ni le temps d'exécution des calculs. L'opérateur $(1+L)^{1 / 2}$ est alors approché de façon plus précise et le résultat des calculs s'en trouve amélioré. La méthode \{fraction quelconque de degré $N+$ Crank-Nicholson $\}$ ne possède pas cette propriété : lors de la résolution d'un problème donné, il n'est pas possible de compenser l'augmentation du degré $N$ de la fraction rationnelle par une augmentation du pas d'intégration selon la distance et de maintenir la précision des calculs sans augmenter de la charge de calcul.

\section{Conclusion.}

Toutes les fois qu'on devra choisir le degré $N$ plus grand que l'unité (approximation parabolique grand angle ou en présence d'un milieu élastique (Collins [5])), on choisira impérativement l'approximation de Bamberger et al. [4] et la méthode des directions alternées implicite. On sait maintenant que ce choix n'est pas forcément pénalisé par un alourdissement des calculs. Le choix de la méthode classique (Crank-Nicholson) est réservé aux cas où $N=1$ : ce choix reste répandu, notamment pour la résolution de l'équation de Tappert.

\section{Références}

[1] J. Piraux, "Equation d'onde parabolique : approximations d'ordre élevé.", Deuxième Congrès Français d'Acoustique, Ed. Journal de Physique, Volume II, 1045-1048 (1992).

[2] G.H. Knightly, D. Lee, D.F. St. Mary, "A higher-order parabolic wave equation", J. Acoust. Soc. Am. 82, 580-587 (1987).

[3] E.H. Vefring, S. Mjølsnes, "A parabolic wave equation based on a rational-cubic approximation", J. Acoust. Soc. Am. 87, 619-623 (1990).

[4] A. Bamberger, B. Engquist, L. Halpern, P. Joly, "Higher order paraxial wave equation approximations in heterogeneous media", SIAM J. Appl. Math. 48, 129-154 (1988).

[5] M.D. Collins, "Higher-order parabolic approximations for accurate and stable elastic parabolic equations with application to interface wave propagation", J. Acoust. Soc. Am. 89, 1050-1057 (1991). 